\title{
小鼠肿瘦及正常器官微血管早期 光动力学损伤的超微结构研究
}

\author{
池顺姬周传农
}

(中国医学科学院肺㴼研究所电子显微镜室, 北京 100021)

\section{关勧词光动力学治坾、小鼠血管、内毛细血管层}

光动力学疗法是一种新的肿瘤治疗手段,近年来发展迅速,受到广泛重视. 但其肿瘦杀伤 的体内作用机理尚未完全阐明. 许多研究结果表明,光动力学治疗后肿痹坏死主要是由于血管 损伤引起的组织缺氧所致 ${ }^{(1)}$. 光动力学作用后, 早期常可见到明显的血管改变、血流减缓以至 完全停滞 ${ }^{[2]}$. 但目前对发生这种变化的原因所知甚少. 本文选用小鼠肝癌 (Hep A) 与 LA-795 肺腺癌两种肿瘤和皮肤、脑、肾、肾上腺四种正常器官, 电镜观察它们经光动力学作用后的超 微结构改变,着重观察光昭后即刻发生的血管内皮细胞的内毛细血管层 (endocapillary layer) 变化, 进一步探讨其损伤与光动力学作用后早期血流改变之间的关系, 阐明光动力学治疗的体 内作用机理, 并为临床上提高光动力学治疗肿瘤的疗效及保护正常器官、减少副作用提供有益 的实验依据.

\section{一、材料与方法}

皮下接种肝癌与肺腺癌的两种荷瘤小鼠及健康 $\mathrm{Balb} / \mathrm{c}$ 小鼠, 尾静脉注射血卟林衍生物 (扬州生化制药厂生产) $20 \mathrm{mg} / \mathrm{kg}$ 体重, $24 \mathrm{~h}$ 后用红光(波长 $600-700 \mathrm{~nm}$, 功率密度 $250 \mathrm{~mW} /$ $\mathrm{cm}^{2}$ ) 炤射肿瘦或有关器官. 两种肿㽷和皮肤的照光剂量为 $50 、 150$ 及 $300 \mathrm{~J} / \mathrm{cm}^{2}$, 脑、肾、肾上 腺的照射剂量为 100 和 $200 \mathrm{~J} / \mathrm{cm}^{2}$. 所有动物均于照光结束后即刻麻醉, 经主动脉或左心室灌 注含 $1 \%$ 硝酸铜的伐二酫与多聚甲醛混合固定液 ( $\mathrm{pH} 7.2$ ) 以显示内毛细血管层. 照光 $150 \mathrm{~J} /$ $\mathrm{cm}^{2}$ 的肝癌小鼠, 还增设了照光后分别于 $1 、 3 、 12$ 及 $24 \mathrm{~h}$ 再做灌注固定的实验组. 灌注后分 别取材, 经酫类与四氧化钱双固定, 环氧树酯 Epon-812 包埋, 超薄切片经醋酸双氧铀与硝酸 铅双重染色, Philips EM-410 型透射电镜观察.

\section{二、观苏结果与讨论}

对炤组肿瘤及正常器官的超微结构均保存良好, 但各种组织的血管内皮细胞表面铜染物 质的分布情况有所不同. 两种肿㿔、皮肤及脑的毛细血管内皮细胞的内毛细血管层铜染阳性, 厚而连续(图 1、3). 肾与肾上腺只有约半数血管的内皮细胞呈锎染阳性.且较薄. 肾小球血管 的内皮细胞表面基本无铜染. 这一结果可能提示: 不同器官中具有不同形态结构与代谢功能

本文 1989 年 11 月 21 日收到. 1990 年 3 月 12 日收到修改稿. 


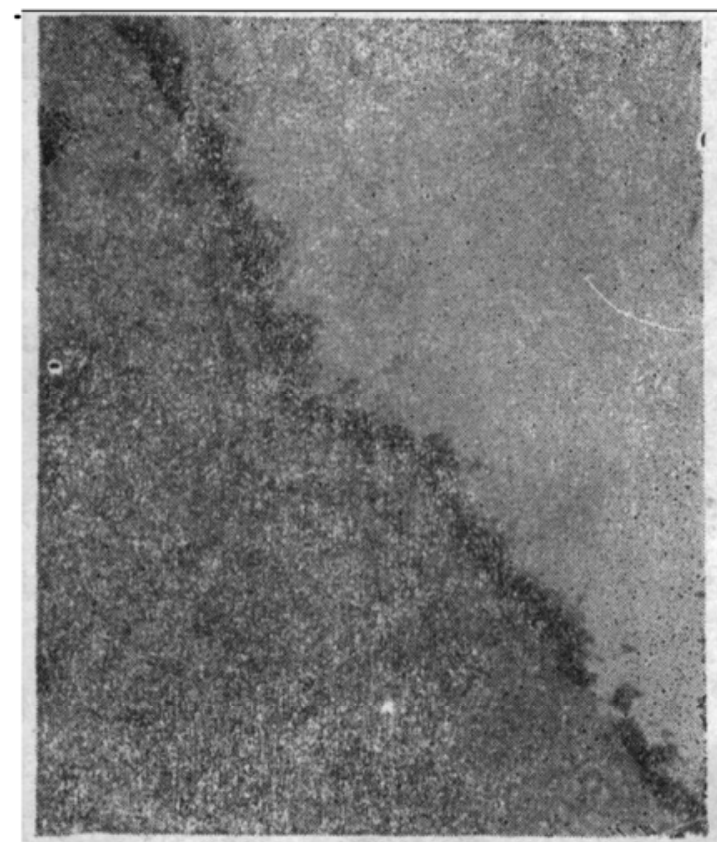

图 1 开癌对嵟组毛细血䇾, 内毛细血管 层锎染阳性, 厚而连续。 $\times 7100$

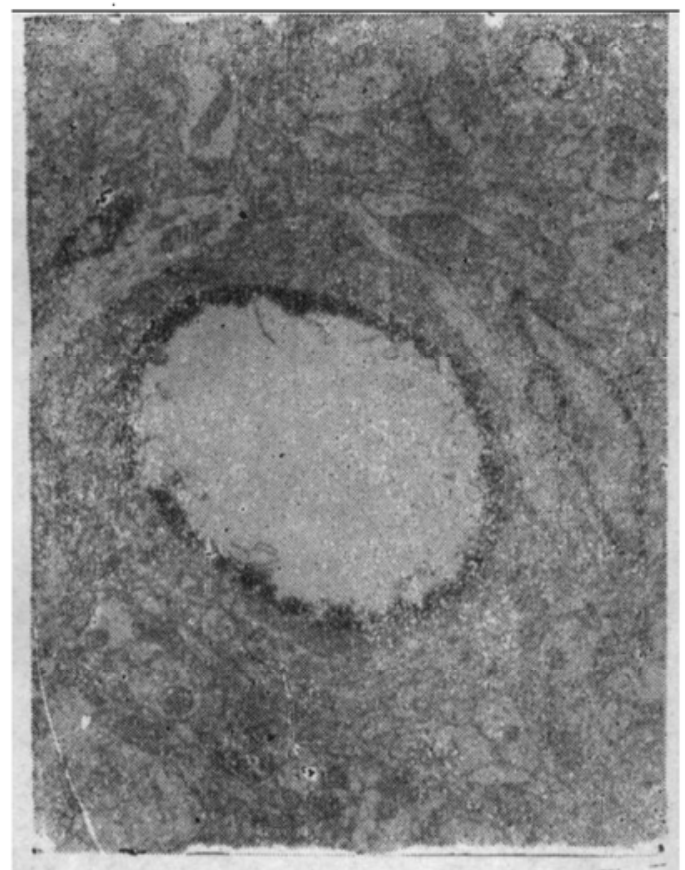

图 3 止常脑单继照光组毛细血管、队正 细伅管层锞染阳性, 厚而连续. $\times 7100$

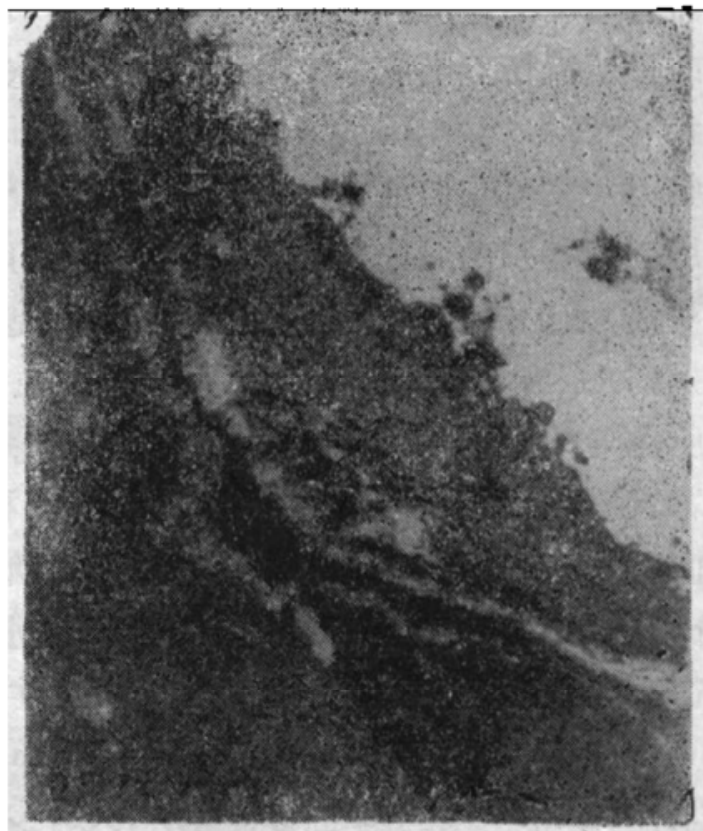

图 2 肝癌光动力学治茫 $50 \mathrm{~J} / \mathrm{cm}^{2}$ 后即刻, 毛细血管的内毛 细血管层基本消失,表面残存少数钢杂斑点. $\times 7100$

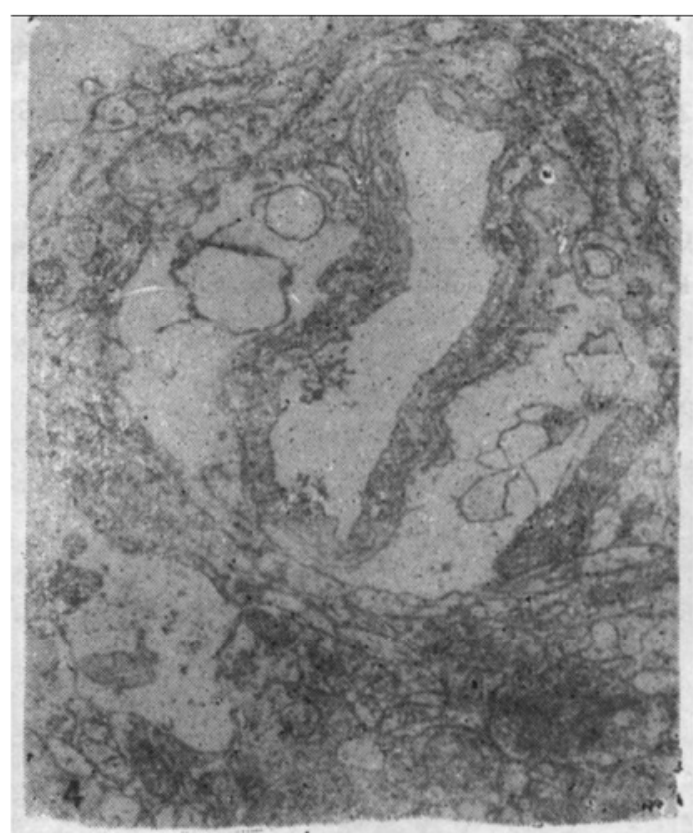

图 4 正常脑光动力学治庁 $200 \mathrm{~J} / \mathrm{cm}^{2}$ 后即刻, 污

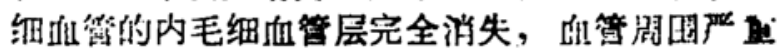
水肿,腔内可见铸䞡沉腚, 附近神经组织无明显改

变. $\times 12000$

特点的毛细血管, 其内皮细胞表面内毛细血管层的组成与电荷分布可能有较大差异.

光动力学作用后即刻, 在所有肿瘦及正常器宣中均未见到实质细胞的损伤改变, 但毛细血 管损伤都很明显. 主要表现为内毛细血管层消失、线粒体肿胀及空泡化，胞质水肿等（图 $2 、$ 4). 较小剂量 $\left(50 \mathrm{~J} / \mathrm{cm}^{2}\right)$ 照光后, 常常仅见内毛细血管层的损伤, 即内皮细胞表面铜染阳些 结构消失. 再次证实血管内皮细胞是体内光动力学作用的主要鞄部位, 并说明其内毛细如管 对光动力学作用尤为㪟感。 
血管内皮细胞的内毛细血管层及其所带负电荷对维持血管内正常血流具有重要意义.内口 毛细血管层损伤, 内皮细胞表面负电荷消失, 可导致红细胞边集及血管内血流意乱 ${ }^{[31}$. 本文 观察到的光动力学作用后内毛细血管层的即刻损伤脱落, 对解释光动力学作用后早期在肿瘦 及某些器官发生的血流紊乱、血栓形成及血管内高血凝状态, 可能具有重要意义. Povlishock 等人用苂光素和光炤处理猫脑血管, 观察到照光后血管内迅速出现血小板烧集; 同时进行超微 结构观察, 只见内皮细胞胞质空泡形成, 细胞膜损伤破裂等改变, 而无血管内皮细胞脱落或血 小板损伤. 因而他们认为, 光照后出现的血小板凝集是由内皮细胞本身的某些损伤引起的 ${ }^{[13}$. 本文所见与他们的结论相符.

本文还观察到, 在光动力学作用后的各种组织中, 小动脉内皮细胞的损伤均较微小静脉和 毛细血管内皮细胞的损伤轻. 其内毛细血管层常仍呈钢染阳性, 厚而连续. 说明小动脉内皮 细胞比较耐受光动力学作用. 其原因尚待进一步探讨.

在肝癌照光 $150 \mathrm{~J} / \mathrm{cm}^{2}$ 后 1-12h 各组中,多数血管的内毛细血管层消失,内皮细胞的损伤 随时间的推移而逐渐加重. 但在照光后 $24 \mathrm{~h}$ 组, 约半数血管的内皮细胞损伤不明显, 表面出现 䥺染阳性, 厚而连续的内毛细血管层, 只有少数血管的改变仍较显著. 这一结果表明, 在光动 力学治疗时, 如光照剂量不足, 血管内皮细胞仅受到较轻、可逆的损伤, 经一定时间后得以修 复. 这一情况可能与光动力学治疗后肿瘤复发有关.

\section{参卒文献}

[1] Zhou, C. N., J. Photochem. Photobiol., B:Biol., 3(1989), 299-318.

[2] Star, W. M. et al. in Photodynamic Therapy of Tumors and Other Diseases (Eds. Jori, G. and Perria, C.), Libreria Progetto, Padova, 1985, 239-242.

[3] Chaudhuri, K. et al., Photochem. Photobiol., 46(1987), 823-827.

[4] Povlishock, J. T. et 2i., Am. J. Pathol., 110(1983), 148-160. 